\title{
Supporting Rapid Product Development with Sketch-Based Modeling
}

\author{
Natthavika Chansri ${ }^{1}$ and Pisut Koomsap ${ }^{2, *}$ \\ ${ }^{1}$ Industrial Engineering, Faculty of Engineering at Si Racha \\ Kasetsart University, Chonburi, Thailand, 20230 \\ natthavika.c@ku.ac.th \\ ${ }^{2}$ Industrial and Manufacturing Engineering, School of Engineering and Technology \\ Asian Institute of Technology, Pathumthani, Thailand, 12120 \\ pisuteait.asia
}

\begin{abstract}
Nowaday, rapid product development (RPD) becomes a crucial approach for the success of a company in a competitive market when the complexity and diversity of products keep increasing while their lifetimes are getting shorter and shorter. Freehand sketch is a tool that is closer and more practical for customer to communicate his/her ideas to manufacturer. The sketch is typically transformed to be a 3D model for technologies such as rapid prototyping where a physical prototype is fabricated from a 3D model. Presented in this paper is a review of sketch-based modeling that allows customers-manufacturers to speed up the idea realization. Sketch-based modeling has been developed not only to transform a sketch to be a 3D model but also to interface with rapid prototyping.
\end{abstract}

Keywords: Rapid product development, Rapid prototyping, Paper-based overtraced freehand sketch, Single line drawing, Sketch-based modeling.

\section{Introduction}

Technologies and tools play significant role in easing the collaboration, and in helping members from different disciplines, who share their expertise to come up with a product, to stimulate and to rapidly realize their ideas. These cover from a basic tool such as a paper and pencil to recent technologies such as computer supported cooperative work or rapid prototyping. Five core technology areas supporting rapid product development are knowledge management, tele-engineering, man machine interface, rapid prototyping, and rapid tooling as shown in Fig. 1 [1].

A prototype is also necessary in product design and development for gaining design insight and for making communication more effective, and prototyping technologies help speed up the process. It can be manufactured quickly with rapid prototyping (RP) technology that allows designer-customer realize and experience their ideas quickly. In RP process, a physical prototype is constructed without using molds and dies. A 3D CAD model is translated into a stack of 2D contours which are used to

\footnotetext{
* Corresponding author.
} 
generate machine commands for constructing a prototype layer by layer. Since commercial CAD software use different data formats, the created model will be converted first to a common format, called Stereolithographic (STL) file. The stack of contours representing constructed layers is obtained from slicing the STL model. Post processes may be required depended upon the selected RP technique. SLA, SLS, LOM, FDM and 3D printing are examples of commercially available RP systems. RP concept has been extended further to rapid tooling [2] and rapid manufacturing [3] for rapid creation of tools and of final products respectively.

Besides creating a 3D CAD model on commercial software, today's technologies makes it happens for the 3D CAD model to be created from its physical object as well as engineering drawing when they are available. Manufacturer can use reverse engineering, RE, to reconstruct a CAD model directly from a product that customer brings in. By coupling RE with RP its physical prototype can be delivered back to customer in a short period of time. Several researchers have tried to improve and to realign steps in both processes to make their direct integration work more effectively [4-5]. The similar idea has been extended to reconstruction of a physical prototype directly from orthographic views drawing, used universally for a long time for communicating designer's ideas to part manufacturing on a shop floor [6].

Another media that is closer and more practical for customer to communicate his/her ideas to manufacturer is a sketch. Freehand sketch is a quick rough drawing for portraying ideas, and commonly used during the conversation to help make ideas easier

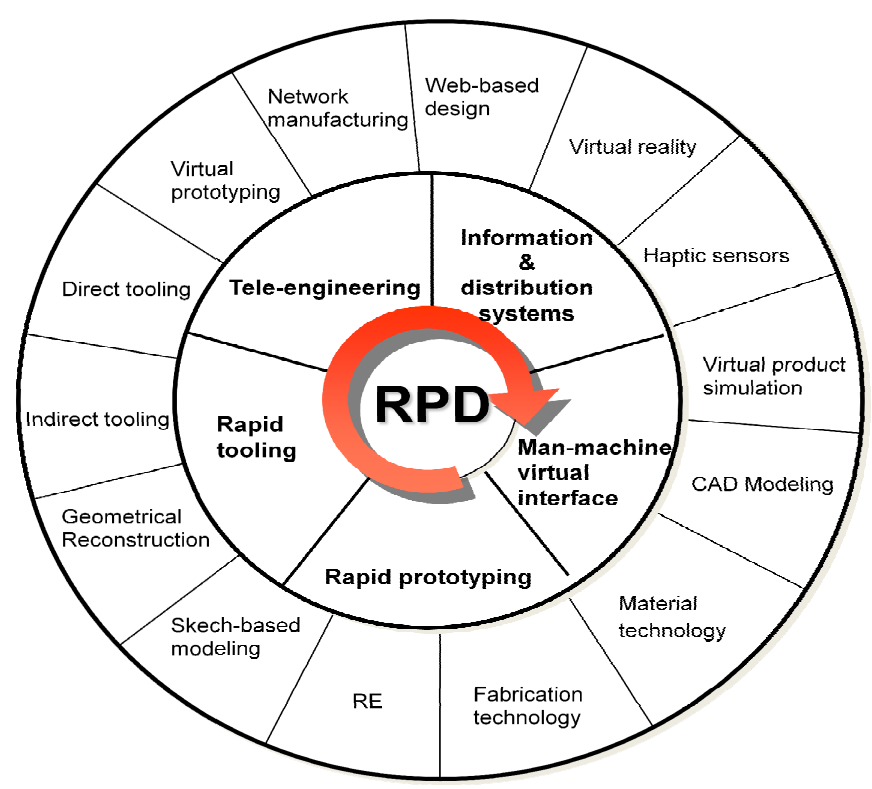

Fig. 1. Rapid product development (modified from [6]) 
to be understood. Furthermore, it is a good interactive media where participants can get involve making correction or revision by drawing together during discussion [7]. Since a freehand sketch is commonly found in practice, a success of its sketch-based modeling will open up several opportunities. It will ease communication and allow customer to visualize clearly the idea. Further extension to direct integrating with rapid prototyping will even make significant impact on product development. Several ideas can be realized and explored. Both customer and manufacturer can gain insight quickly not only from a virtual prototype but also a physical one. Product development time is foreseen to be shortened drastically. Presented in this paper is a review of sketch-based modeling that allows not only a 3D model to be reconstructed from a sketch but also a physical prototype to be fabricated from the 3D model directly to be realized rapidly. The next section presents sketch-based modeling, followed by interfacing sketch-based modeling with RP. The conclusion is addressed in the last section.

\section{$2 \quad$ Sketch-Based Modeling}

Sketch-based modeling, SBM, has been introduced for a CAD model reconstruction from a freehand sketch. Several approaches have been proposed and they can be classified into two main groups: evocative-based 3D modeling and constructive-based 3D modeling [8]. Based on prior knowledge, evocative-based modeling reconstructs a 3D model from a set of primitive objects or templates that is pre-registered in a database. A pattern of intended strokes is compared with registered patterns, and the information of the matched pattern is retrieved. Two main approaches for evocative-based 3D modeling are iconic [9], and template retrieval [10]. In case of constructive-based modeling, a 3D model is reconstructed systematically by applying visual rules on obtained sketch information. Two main approaches are optimization and progressive. For optimization approach, the depth information of an object is determined from an objective function that is constrained by geometrical regularity rules [11-13]. Instead of identifying 3D coordinates of all vertices simultaneously, the progressive approach determines the coordinates of the vertices in order by propagation [14-16].

Illustrated in Fig. 2 is the general constructive-based 3D modeling steps taken for 3D model reconstruction from a 2D freehand sketch that can be classified into four groups: a non-overtraced online freehand sketch, an overtraced online freehand sketch, a non-overtraced offline freehand sketch, and an overtraced offline freehand sketch. Typically, a sketch is converted to be a single-line drawing which can be considered as the projected image of a 3D object on a plane from where junction points, segments and junction loops can be extracted, and used to determine vertices, edges and faces for recreation of the model in a 3D space. The 3D model is then reconstructed after 3D coordinates of all vertices become available. The details are presented in the following subsections. 


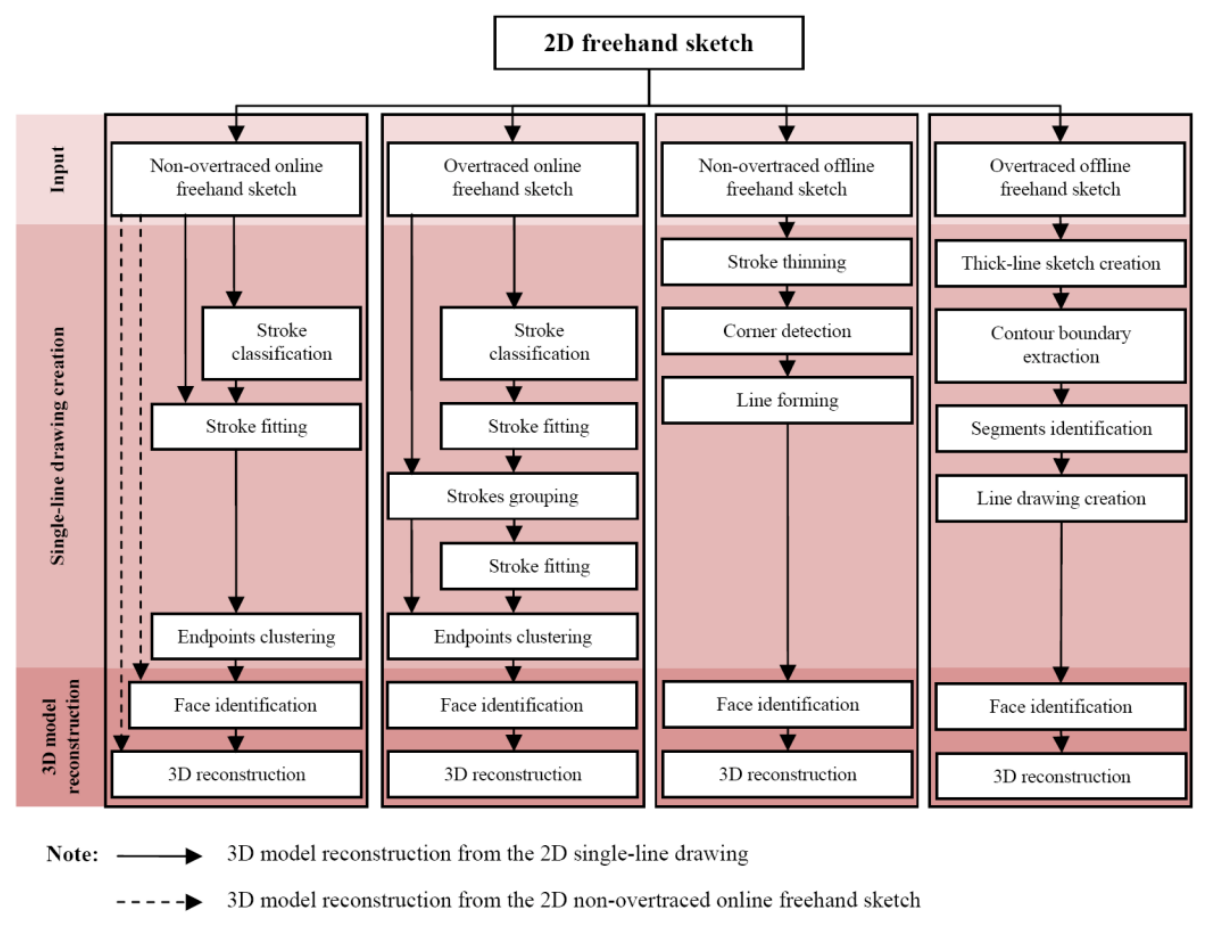

Fig. 2. Overview for constructive-based 3D modeling process from $2 \mathrm{D}$ freehand sketch (modified from [21])

\subsection{Single-Line Drawing Creation}

As aforementioned, besides Kang et al. [12] and Masry et al. [13] that construct a 3D model directly from a 2D non-overtraced online sketch, most of the constructivebased modeling techniques start with creating a single-line drawing from a sketch. In case of a non-overtraced online sketch, obtaining a single-line drawing is considered to be the simplest one as each stroke represents a segment on an intended sketch, and stroke information, including their two endpoints, is available for all strokes [17]. Usually, these strokes are beautified before their endpoints are connected to form a single-line drawing. In case of an overtraced online sketch that the data on each stroke are arranged in order while the entire set of data representing overlapping strokes is unordered, additional steps are required to group overtraced strokes and to determine their representatives [15]. In case of a non-overtraced offline sketch that data are available as a batch of point in a scanned sketch image from which these points represent closed contours of single solid lines, they must be extracted before their endpoints are linked to form a single-line drawing [18-20]. In case of an overtraced freehand sketch, Chansri and Koomsap [21] have recently introduced an approach on identifying a single-line drawing from a paper-based overtraced freehand sketch. In their approach, image processing techniques have been applied to cluster strokes to form a thick-line sketch. Contour expanding and shrinking concept has been applied 
in this approach for identifying segments from every pair of extracted contours that are said to be neighbor. A single-line drawing is created after all junctions are identified.

\subsection{D Model Reconstruction}

In this step, faces are identified from the obtained single-line drawing. Line labeling is a popular technique that works well for identifying faces from a single-line drawing with hidden lines free where all identified non-self-intersecting closed loops represent actual faces $[14,18]$. Line labeling, however, may not be appropriate for a wireframe drawing that some of non-self-intersecting closed loops do not represent actual faces. Possible faces that are checked for their coexisting have been introduced to identify actual faces on the wireframe drawing [11, 22]. Besides, there is an attempt to identify faces from a 2D freehand sketch directly [23]. After all faces are obtained, the $3 \mathrm{D}$ model is reconstructed by using one of the two approaches as mentioned earlier.

\section{Rapid Prototyping and Its Interfacing with Sketch-Based Modeling}

Reverse engineering and sketch-based modeling are in common that they all give a 3D CAD model as an output, which can be an input for rapid prototyping. Their direct integration with RP will allow quick creation of a physical prototype from its physical object and freehand sketch. However, attempts toward rapid prototyping have been different. Fig.3 illustrates existing links between RE and SBM with RP.

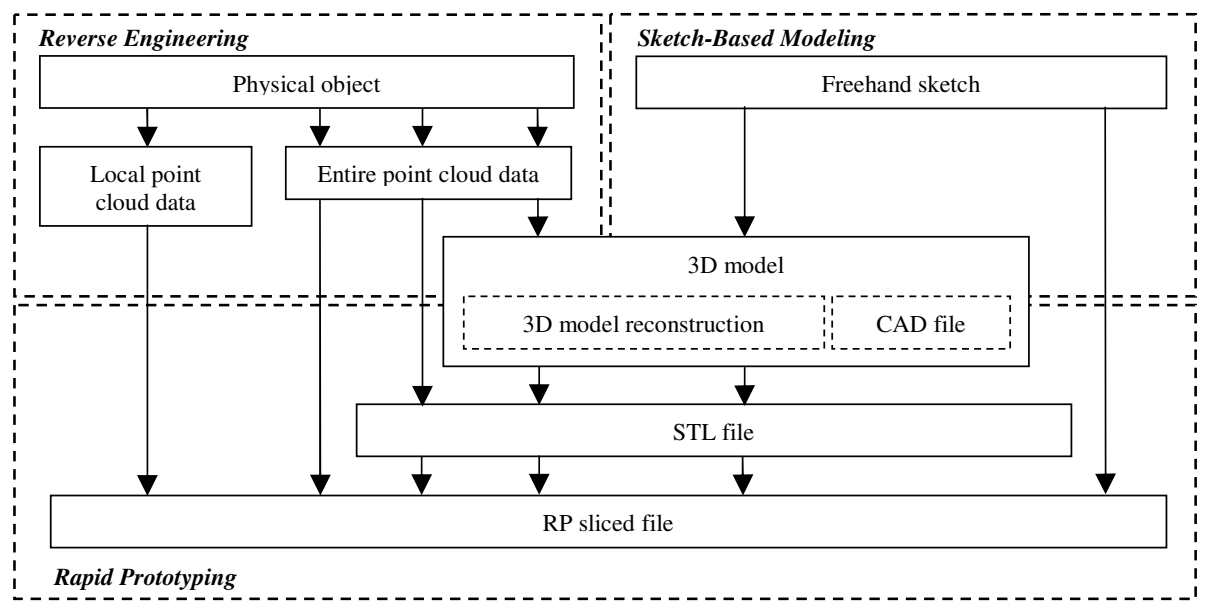

Fig. 3. RE-RP interface and SBM-RP interface 
For reverse engineering, research is not limited to 3D CAD model reconstruction. Several researchers have tried to strengthen and make a connection between RE and RP more effective. It can be classified into four approaches. The first approach is commonly found in practice. Point cloud data are acquired from an object and gone through regular RE process to reconstruct a 3D CAD model. The obtained model is then fed into RP process. However because surface reconstruction consumes $90-95 \%$ of the RE processing time comparing to 5-10\% of that for digitizing physical prototype [24], it is skipped in the second approach where an STL file is created directly from the point cloud [25]. Inherent errors from STL conversion process have inspired the third approach where commands are generated directly from the point cloud [4]. For these three approaches, however, the entire surface data come in a large batch with data redundancy that need to be removed to simplify subsequent operations. Selective data acquisition has been introduced as an alternative for direct interfacing RE with RP [26]. Instead of acquiring entire point cloud data, it scans an object selectively from the bottom up according to the complexity of the object. The scanning result appears as a stack of contours which can be directly used to generate machine commands.

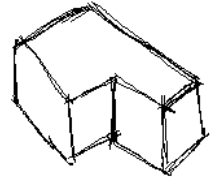

A paper-based overtraced freehand sketch
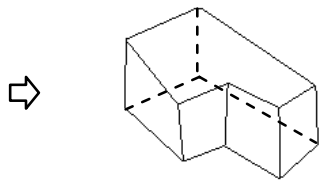

A single-line drawing with its $3 \mathrm{D}$ coordinates

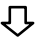

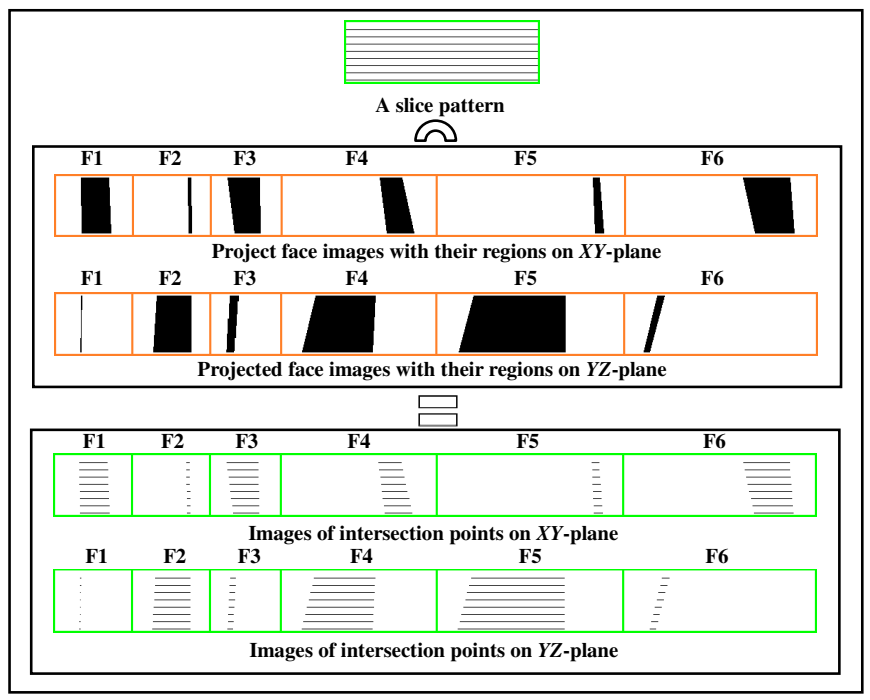

Preparing a slice pattern and slicing face images

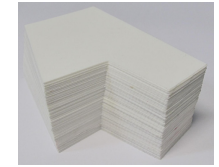

A physical prototype

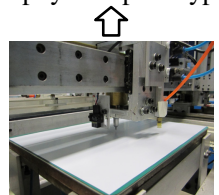

RP process ษ

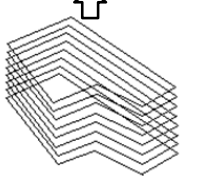

A stack of contours

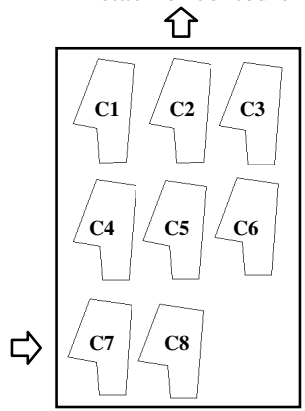

Creating contours

Fig. 4. Overview of direct interfacing sketch-based modeling with rapid prototyping 
Similar concept has been applied to create direct integration between sketch-based modeling and rapid prototyping, instead of leaving a sketch-based modeling be a stand-alone technology. Recently, image-based direct slicing has been introduced as a first attempt for direct interfacing sketch-based modeling with RP [27]. This approach composes of three main steps: preparing a slice pattern, slicing face images, and constructing a stack of contours. The input to this approach is an isometric single-line drawing and its projected face images on the vertical principal planes. The process starts with projecting all faces obtained during identifying the 3D coordinates of vertices [16] on their principal vertical planes where uniform direct slicing is conducted layer by layer simultaneously on these planes. Segments, obtained from the faces, are connected to form a closed contour for each layer. The output is a stack of closed contours that will be used to generate commands directly for RP fabrication process. Fig. 4 illustrates the overview of direct interfacing sketch-based modeling with rapid prototyping.

\section{Conclusions}

This paper presents a review of sketch-based modeling to support rapid product development. Sketch-based modeling has been developed that allows a 3D model to be reconstructed from a sketch and a physical prototype to be fabricated from a 3D model. However, except for using pre-defined information as in evocative-based 3D modeling approarch, sketch-based modeling is limited to an object that composes of only planar surfaces. The contours generation for rapid prototyping needs further study to handle the object with curve surfaces.

\section{References}

1. Bernard, A., Fischer, A.: New trends in rapid product development. CIRP Annals- Manufacturing Technology 51(2), 635-652 (2002)

2. Radstok, E.: Rapid tooling. Rapid Prototyping Journal 5(4), 164-168 (1999)

3. Levy, G.N., Schindel, R., Kruth, J.P.: Rapid manufacturing and rapid tooling with layer manufacturing (LM) technologies, state of the art and future perspectives. CIRP AnnalsManufacturing Technology 52, 589-609 (2003)

4. Lee, K.H., Woo, H.: Direct integration of reverse engineering and rapid prototyping. Computers \& Industrial Engineering 38(1), 21-38 (2000)

5. Liu, G.H., Wong, Y.S., Zhang, Y.F., Loh, H.T.: Modeling cloud data for prototype manufacturing. J. Mater. Process. Technol. 38, 53-57 (2003)

6. Soonanon, P., Koomsap, P.: Towards direct transformation of orthographic-view drawings into a prototype. Virtual and Physical Prototyping 4(2), 75-90 (2009)

7. Company, P., Contero, M., Varley, P., Aleixos, N., Naya, F.: Computer-aided sketching as a tool to promote innovation in the new product development process. Computers in Industry 60(8), 592-603 (2009)

8. Olsen, L., Samavati, F.F., Sousa, M.C., Jorge, J.A.: Sketch-based modeling: A survey. Comput. Graph 33(1), 85-103 (2009) 
9. Shesh, A., Chen, B.: SMARTPAPER: an interactive and user friendly sketching system, Comput. Graph Forum 23(3), 301-310 (2004)

10. Cheon, S.U., Han, S.H.: A template-based reconstruction of plane-symmetric 3D models from freehand sketches. Computer-Aided Design 40(9), 975-986 (2008)

11. Shpitalni, M., Lipson, H.: Identification of faces in a 2D line drawing projection of a wireframe object. IEEE Transactions on Pattern Analysis and Machine Intelligence 18(10), 1000-1012 (1996)

12. Kang, D., Masry, M., Lipson, H.: Reconstruction of a 3D object from a main axis system. In: American Association for Artificial Intelligence Fall Symposium on making Pen-based Interaction Intelligent and Nature, pp. 63-69 (2004)

13. Masry, M., Kang, D., Lipson, H.: A freehand sketching interface for progressive construction of 3D objects. Computers \& Graphic 29(4), 563-575 (2005)

14. Lamb, D., Bandopadhay, A.: Interpreting a 3D object from a rough 2D line drawing. In: The First IEEE Conference on Visualization, San Francisco, California, pp. 59-66 (1990)

15. Ku, D.C., Qin, S.F., Wright, D.K.: Interpretation of ovetracing freehand sketching for geometric shapes. In: WSCG 2006, Conference in Central Europe on Computer Graphics, Visualization and Computer Vision, Plzen, Czech Republic, pp. 263-270 (2006)

16. Chansri, N., Koomsap, P., Kuagoolkijgarn, P.: Application of contour tracing algorithm in 3D modeling from a paper-based overtraced freehand sketch. In: The 21st International Conference on Flexible Automation and Intelligent Manufacturing, Taichung, Taiwan (2011)

17. Shpitalni, M., Lipson, H.: Classification of sketch strokes and corner detection using conic sections and adaptive clustering. Journal of Mechanical Design 119, 131-135 (1995)

18. Marti, E., Regincos, J., Lopez-Krahe, J., Villanueva, J.J.: Line drawing interpretation as polyhedral objects to man-machine interaction in CAD systems. Pattern Recognition and Image Analysis, 1-12 (1995)

19. Haron, H., Mohamed, D., Shamsuddin, S.M.: Extraction of junctions, lines and regions of irregular line drawing: the chain code processing algorithm. Jurnal Teknologi 38(D), 1-28 (2003)

20. Farrugia, P.J., Borg, J.C., Camilleri, K.P., Spiteri, C., Bartolo, A.: A cameraphone-based approach for the generation of 3D models from paper sketches. In: EUROGRAPHICS Workshop on Sketch-Based Interfaces and Modeling, pp. 33-42 (2004)

21. Chansri, N., Koomsap, P.: Automatic single-line drawing creation from a paper-based overtraced freehand sketch. Int. J. Adv. Manuf. Technol. 59(1-4), 221-242 (2011)

22. Liu, J.Z., Lee, Y.T.: A graph-based method for face identification from a single 2D line drawing. IEEE Transactions on Pattern Analysis and Machine Intelligence 23(10), 11061119 (2001)

23. Oh, B.S., Kim, C.H.: Progressive reconstruction of $3 \mathrm{D}$ objects from a single freehand line drawing. Computers \& Graphics 27(4), 581-592 (2003)

24. Schoene, C., Hoffmann, J.: Reverse Engineering based on multi-axis digitizer data. In: International Conference on Manufacturing Automation (ICMA 1997), pp. 909-914 (1997)

25. Liu, Z., Wang, L., Lu, B.: Integrating cross-sectional imaging based reverse engineering with rapid prototyping. Computers in Industry 57(2), 131-140 (2006)

26. Rianmora, S., Koomsap, P., Hai, D.P.: Selective data acquisition for direct integration of reverse engineering and rapid prototyping. Virtual and Physical Prototyping 4(4), 227-239 (2009)

27. Chansri, N., Koomsap, P.: Image-based direct slicing of a single line drawing for rapid prototyping. In: The 5th International Conference on Advanced Research in Virtual and Rapid Prototyping, Leiria, Portugal (2011) 